\title{
Erratum to: Dehydrogenation of 2-Phenyl-1-pyrroline with Palladium-Supported Catalysts: An Effective Route to the Synthesis of 2-Phenylpyrrole
}

\author{
Cláudia A. Figueira ${ }^{1} \cdot$ Pedro T. Gomes ${ }^{1}$
}

Published online: 24 March 2015

(c) Springer Science+Business Media New York 2015

\section{Erratum to: Catal Lett (2015) 145:762-768 \\ DOI 10.1007/s10562-014-1469-0}

The original version of this article unfortunately contained an error in Table 1. The unit mentioned in the header of last column in Table 1 (TOF) should be changed to $\min ^{-1}$ instead of $\mathrm{s}^{-1}$. The correct table is given below. doi:10.1007/s10562-014-1469-0.

$\triangle$ Pedro T. Gomes

pedro.t.gomes@tecnico.ulisboa.pt

1 Centro de Química Estrutural, Departamento de Engenharia Química, Instituto Superior Técnico, Universidade de Lisboa, Av. Rovisco Pais, 1, 1049-001 Lisboa, Portugal 
Table 1 Reaction data (conversions, yields and selectivities) obtained for the catalytic dehydrogenation of 2-phenyl-1-pyrroline to 2-phenylpyrrole with Pd-supported catalysts

\begin{tabular}{|c|c|c|c|c|c|c|c|c|}
\hline $\begin{array}{l}\text { Reaction } \\
\text { no. }\end{array}$ & $\begin{array}{l}\text { Catalytic } \\
\text { Support }^{\mathrm{a}}\end{array}$ & Supplier & $\begin{array}{l}\mathrm{Pd}^{\mathrm{b}} \\
(\mathrm{mol} \%)\end{array}$ & $\begin{array}{l}\text { Time } \\
\text { (h) }\end{array}$ & $\begin{array}{l}\text { Conv. }{ }^{\mathrm{c}} \\
(\%)\end{array}$ & $\begin{array}{l}\text { Yield }^{\mathrm{d}} \\
(\%)\end{array}$ & $\begin{array}{l}\text { Select. }{ }^{\mathrm{e}} \\
(\%)\end{array}$ & $\begin{array}{l}\operatorname{TOF}^{\mathrm{f}} \\
\left(\mathrm{min}^{-1}\right)\end{array}$ \\
\hline $\mathrm{C} 1^{\mathrm{g}}$ & $\mathrm{C}$ & Merck & 10.00 & 115 & 42 & 42 & 100 & 0.001 \\
\hline $\mathrm{C} 2$ & $\mathrm{C}$ & Merck & 10.00 & 3.0 & 94 & 81 & 86 & 0.056 \\
\hline $\mathrm{C} 3$ & $\mathrm{C}$ & Acrös & 1.25 & 5.8 & 92 & 78 & 85 & 0.230 \\
\hline $\mathrm{C} 4$ & $\mathrm{C}$ & Merck & 1.00 & 4.5 & 94 & 81 & 86 & 0.370 \\
\hline C5 & $\mathrm{C}$ & Acrös & 0.40 & 28 & 86 & 77 & 90 & 0.149 \\
\hline C6 & $\mathrm{C}$ & Acrös & 0.10 & 43 & 52 & 49 & 93 & 0.388 \\
\hline Al1 & $\mathrm{Al}_{2} \mathrm{O}_{3}$ & Fluka & 1.00 & 1.5 & 97 & 79 & 82 & 1.111 \\
\hline $\mathrm{A} 12$ & $\mathrm{Al}_{2} \mathrm{O}_{3}$ & Fluka & 0.40 & 1.5 & 98 & 90 & 93 & 2.778 \\
\hline $\mathrm{Al} 3$ & $\mathrm{Al}_{2} \mathrm{O}_{3}$ & Fluka & 0.10 & 57 & 76 & 64 & 84 & 0.292 \\
\hline
\end{tabular}

Reaction conditions: $\mathrm{n}_{\text {pyrroline }}=$ initial number of moles of 2-phenyl-1-pyrroline $(13.8 \mathrm{mmol}, 2 \mathrm{~g}) ; \mathrm{V}_{\mathrm{xylene}}=40 \mathrm{ml} ; \mathrm{T}=150{ }^{\circ} \mathrm{C}$

a Supports: activated carbon $(\mathrm{C})$ and alumina $\left(\mathrm{Al}_{2} \mathrm{O}_{3}\right)$

${ }^{\mathrm{b}} \mathrm{mol} \% \mathrm{Pd}=\left[\mathrm{n}_{\mathrm{Pd}} / \mathrm{n}_{\text {pyrroline }}\right] \times 100 ; \mathrm{n}_{\mathrm{Pd}}=$ moles of Pd in the supported catalyst

${ }^{\mathrm{c}}$ Conversion of 2-phenyl-1-pyrroline (determined by ${ }^{1} \mathrm{H}$ NMR spectroscopy)

${ }^{\mathrm{d}}$ Yield in 2-phenylpyrrole (determined by ${ }^{1} \mathrm{H}$ NMR spectroscopy)

e Selectivity in 2-phenylpyrrole (determined by ${ }^{1} \mathrm{H}$ NMR spectroscopy)

${ }^{\mathrm{f}} \mathrm{TOF}=$ turnover frequency $=$ moles of 2-phenyl-1-pyrroline converted/(moles $\mathrm{Pd} \times$ time $)$

g Reaction conditions: $\mathrm{n}_{\text {pyrroline }}=$ initial number of moles of 2-phenyl-1-pyrroline $(6.89 \mathrm{mmol}, 1 \mathrm{~g}) ; \mathrm{V}_{\text {toluene }}=10 \mathrm{ml} ; \mathrm{T}=120{ }^{\circ} \mathrm{C}$ 